Saralees Nadarajah (Manchester)

SAmuel Kotz (Washington)

\title{
A MULTIMODAL BETA DISTRIBUTION WITH APPLICATION TO ECONOMIC DATA
}

Abstract. Beta distributions are popular models for economic data. In this paper, a new multimodal beta distribution with bathtub shaped failure rate function is introduced. Various structural properties of this distribution are derived, including its cdf, moments, mean deviation about the mean, mean deviation about the median, entropy, asymptotic distribution of the extreme order statistics, maximum likelihood estimates and the Fisher information matrix. Finally, an application to consumer price indices is illustrated to show that the proposed distribution is a better model to economic data than one based on the standard beta distribution.

1. Introduction. Beta distributions are very versatile and a variety of uncertainties can be usefully modeled by them. Many of the finite range distributions encountered in practice can be easily transformed into the standard distribution. In economics, many times the data are modeled by finite range distributions.

A random variable $X$ is said to have the standard beta distribution with parameters $\alpha$ and $\beta$ if its probability density function (pdf) is

$$
f(x)=\frac{x^{\alpha-1}(1-x)^{\beta-1}}{B(\alpha, \beta)}
$$

for $0<x<1, \alpha>0$ and $\beta>0$, where

$$
B(a, b)=\int_{0}^{1} t^{a-1}(1-t)^{b-1} d t
$$

denotes the beta function. Many generalizations of (1) involving algebraic,

2000 Mathematics Subject Classification: 33C90, 62E99.

Key words and phrases: beta distribution. 
exponential and hypergeometric functions have been proposed in the literature; see Chapter 25 in Johnson et al. (1995) and Gupta and Nadarajah (2004) for detailed accounts. In this paper, we introduce a new distribution that contains several of the known generalizations as particular cases. We derive various structural properties of this new distribution, including its cdf, moments, mean deviation about the mean, mean deviation about the median, entropy, asymptotic distribution of the extreme order statistics, maximum likelihood estimates and the Fisher information matrix (Sections 2-8). We also present an application of the proposed model to consumer price indices (Section 9). One of the attractive properties of this new distribution is that its pdf is multimodal and that its failure rate function can be bathtub shaped.

The calculations involve several special functions, including the incomplete beta function defined by

$$
B_{x}(a, b)=\int_{0}^{x} t^{a-1}(1-t)^{b-1} d t,
$$

the Gauss hypergeometric function defined by

$$
{ }_{2} F_{1}(a, b ; c ; x)=\sum_{k=0}^{\infty} \frac{(a)_{k}(b)_{k}}{(c)_{k}} \frac{x^{k}}{k !}
$$

and the Appell function of the first kind defined by

$$
F_{1}(a, b, c ; d ; x, y)=\sum_{m=0}^{\infty} \sum_{n=0}^{\infty} \frac{(a)_{m+n}(b)_{m}(c)_{n} x^{m} y^{n}}{(d)_{m+n} m ! n !},
$$

where $(f)_{k}=f(f+1) \cdots(f+k-1)$ denotes the ascending factorial. The properties of the above special functions can be found in Prudnikov et al. (1986) and Gradshteyn and Ryzhik (2000).

2. Probability density function. We define the new distribution by the pdf

$$
f(x)=\frac{C x^{\alpha-1}(1-x)^{\beta-1}}{(1-u x)^{\varrho}(1-v x)^{\lambda}}
$$

for $0<x<1, \alpha>0, \beta>0, \varrho>0, \lambda>0,-1<u<1$ and $-1<v<1$, where $C$ denotes the normalizing constant. Application of equation (2.2.8.5) in Prudnikov et al. (1986, Volume 1) shows that the normalizing constant is given by

$$
1 / C=B(\alpha, \beta) F_{1}(\alpha, \varrho, \lambda, \alpha+\beta ; u, v)
$$

and thus we refer to $(2)$ as the $F_{1}$ beta distribution. This new distribution is very flexible and it contains several of the known generalizations of (1) as particular cases. The standard beta distribution in (1) is the particular case 
for either $\varrho=0$ and $\lambda=0$ or $\varrho=0$ and $v=0$ or $u=0$ and $\lambda=0$ or $u=0$ and $v=0$. Libby and Novick's (1982) beta distribution is the particular case for either $\varrho=\alpha+\beta$ and $\lambda=0$ or $u=v$ and $\varrho+\lambda=\alpha+\beta$. Armero and Bayarri's (1994) Gauss hypergeometric distribution is the particular case for either $\varrho=0$ or $\lambda=0$.
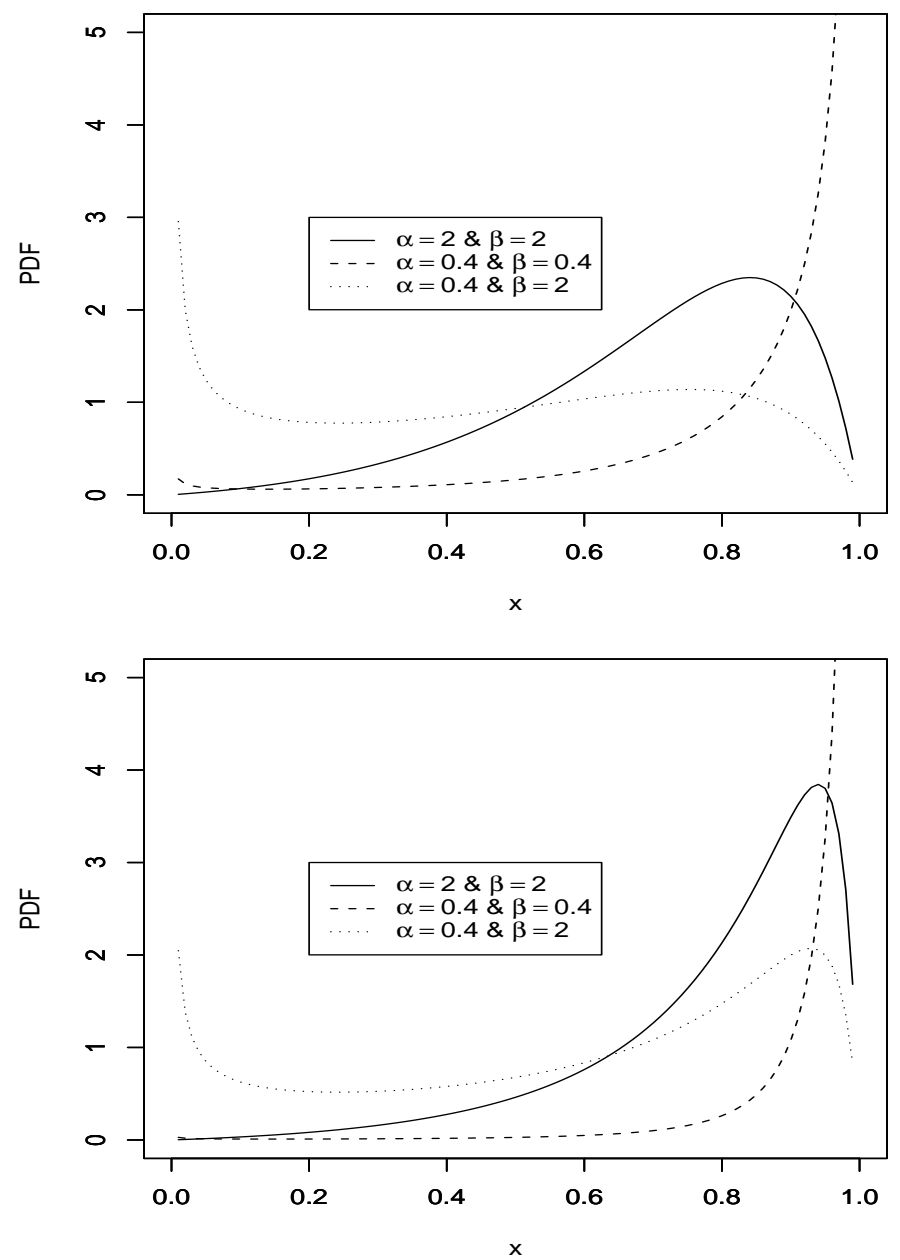

Fig. 1. Plots of the pdf (2) for $u=0.1, v=0.4, \varrho=2.5, \lambda=8$ (top) and $u=0.1, v=0.9$, $\varrho=9, \lambda=2.5$ (bottom)

Let us now consider the shape of (2). The first derivative of $\log f$ is

$$
\frac{d \log f}{d x}=\frac{\alpha-1}{x}-\frac{\beta-1}{1-x}+\frac{\varrho u}{1-u x}+\frac{\lambda v}{1-v x} .
$$

Setting this to zero, one obtains the cubic equation

$$
A x^{3}-B x^{2}-E x-\alpha+1=0,
$$



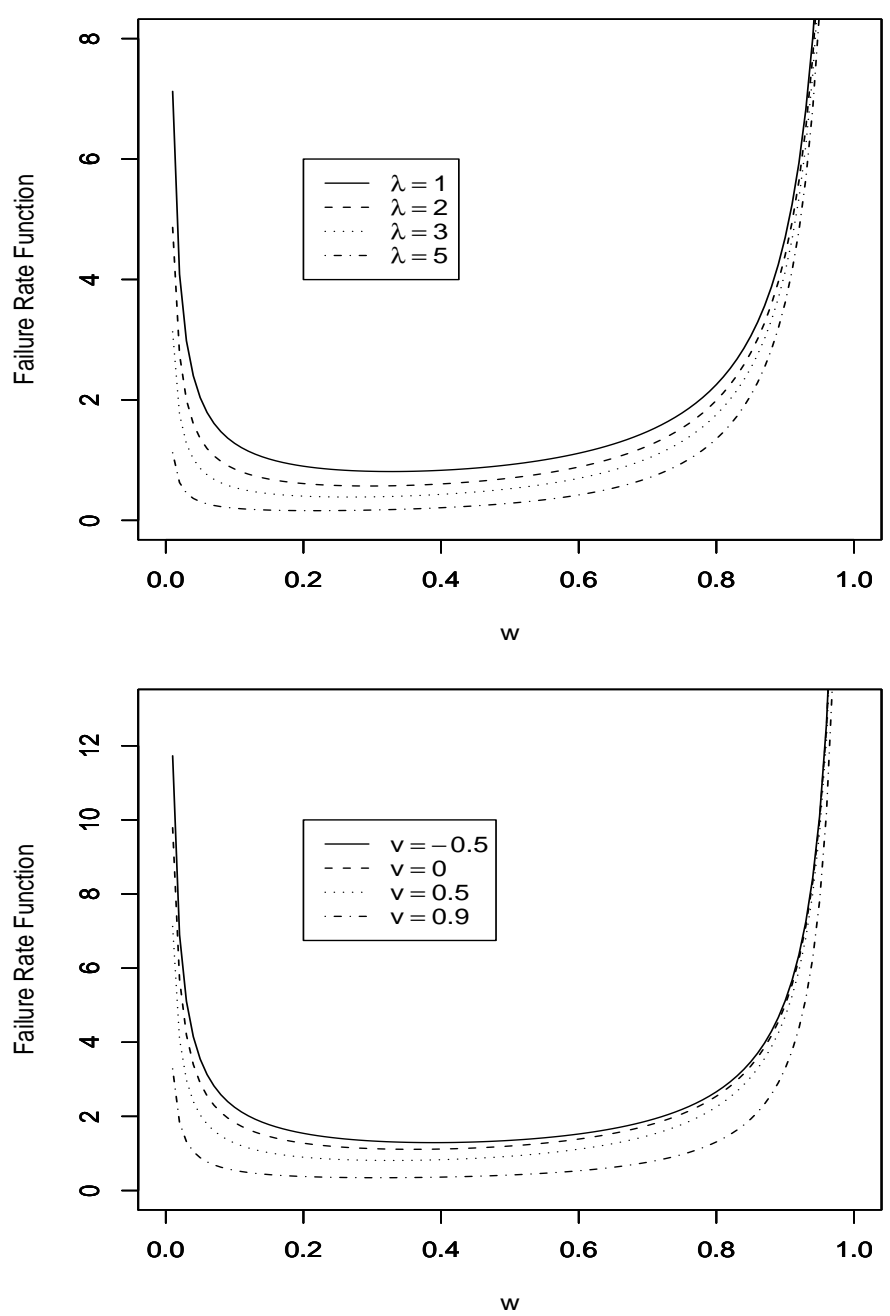

Fig. 2. Plots of the failure rate function of (2) for $\alpha=0.1, \beta=0.5, u=0.5, v=0.5$, $\varrho=1$ (top) and $\alpha=0.1, \beta=0.5, u=0.5, \varrho=1, \lambda=8$ (bottom)

where

$$
\begin{aligned}
& A=u v(\alpha-2+\beta-\varrho-\lambda), \\
& B=u v \alpha-u v+v \alpha-2 v+u \alpha-2 u+v \beta+u \beta-\varrho u v-\varrho u-\lambda u v-\lambda v, \\
& E=-v \alpha+v-u \alpha+u-\alpha+2-\beta+\varrho u+\lambda v .
\end{aligned}
$$

Thus, in principle, the pdf can contain up to three turning points. Some possible shapes with more than one turning point are illustrated in Figure 1 for selected values of $\alpha, \beta, \varrho$ and $\lambda$. Some possible bathtub shapes of the failure rate function $f(x) / \int_{x}^{\infty} f(y) d y$ corresponding to (2) are shown in Figure 2. 
3. Cumulative distribution function. Direct expressions for the cdf of (2) are not possible. Here, we provide four series representations for the cdf. Firstly, using the series expansion

$$
(1-u x)^{-\varrho}=\sum_{j=0}^{\infty}\left(\begin{array}{c}
-\varrho \\
j
\end{array}\right)(-u x)^{j}
$$

one can write

$$
\begin{aligned}
F(x) & =C \int_{0}^{x} y^{\alpha-1}(1-y)^{\beta-1}(1-u y)^{-\varrho}(1-v y)^{-\lambda} d y \\
& =C \int_{0}^{x} y^{\alpha-1}(1-y)^{\beta-1}\left\{\sum_{j=0}^{\infty}\left(\begin{array}{c}
-\varrho \\
j
\end{array}\right)(-u y)^{j}\right\}(1-v y)^{-\lambda} d y \\
& =C \sum_{j=0}^{\infty}\left(\begin{array}{c}
-\varrho \\
j
\end{array}\right)(-u)^{j} \int_{0}^{x} y^{j+\alpha-1}(1-y)^{\beta-1}(1-v y)^{-\lambda} d y \\
& =C x^{\alpha} \sum_{j=0}^{\infty}\left(\begin{array}{c}
-\varrho \\
j
\end{array}\right) \frac{(-u x)^{j}}{\alpha+j} F_{1}(\alpha+j, 1-\beta, \lambda, \alpha+j+1 ; x, v x),
\end{aligned}
$$

where the last step follows by application of equation (2.2.8.5) in Prudnikov et al. (1986, Volume 1). Similarly, using the series expansion

$$
(1-v x)^{-\lambda}=\sum_{j=0}^{\infty}\left(\begin{array}{c}
-\lambda \\
j
\end{array}\right)(-v x)^{j}
$$

one can obtain

$$
F(x)=C x^{\alpha} \sum_{j=0}^{\infty}\left(\begin{array}{c}
-\lambda \\
j
\end{array}\right) \frac{(-v x)^{j}}{\alpha+j} F_{1}(\alpha+j, 1-\beta, \lambda, \alpha+j+1 ; x, u x) .
$$

Thirdly, using both expansions (3) and (4), one can write

$$
\begin{aligned}
F(x) & =C \int_{0}^{x} y^{\alpha-1}(1-y)^{\beta-1}\left\{\sum_{j=0}^{\infty}\left(\begin{array}{c}
-\varrho \\
j
\end{array}\right)(-u y)^{j}\right\}\left\{\sum_{k=0}^{\infty}\left(\begin{array}{c}
-\lambda \\
k
\end{array}\right)(-v y)^{k}\right\} d y \\
& =C \sum_{j=0}^{\infty} \sum_{k=0}^{\infty}\left(\begin{array}{c}
-\varrho \\
j
\end{array}\right)\left(\begin{array}{c}
-\lambda \\
k
\end{array}\right)(-u)^{j}(-v)^{k} \int_{0}^{x} y^{j+k+\alpha-1}(1-y)^{\beta-1} d y \\
& =C \sum_{j=0}^{\infty} \sum_{k=0}^{\infty}\left(\begin{array}{c}
-\varrho \\
j
\end{array}\right)\left(\begin{array}{c}
-\lambda \\
k
\end{array}\right)(-u)^{j}(-v)^{k} B_{x}(\alpha+i+j, \beta),
\end{aligned}
$$

where the last step follows from the definition of the incomplete beta func- 
tion. Finally, using the series expansion

$$
(1-x)^{\beta-1}=\sum_{j=0}^{\infty}\left(\begin{array}{c}
\beta-1 \\
j
\end{array}\right)(-x)^{j},
$$

one can write

$$
\begin{aligned}
F(x) & =C \int_{0}^{x} y^{\alpha-1}\left\{\sum_{j=0}^{\infty}\left(\begin{array}{c}
\beta-1 \\
j
\end{array}\right)(-y)^{j}\right\}(1-u y)^{-\varrho}(1-v y)^{-\lambda} d y \\
& =C \sum_{j=0}^{\infty}\left(\begin{array}{c}
\beta-1 \\
j
\end{array}\right)(-1)^{j} \int_{0}^{x} y^{j+\alpha-1}(1-u y)^{-\varrho}(1-v y)^{-\lambda} d y \\
& =C x^{\alpha} \sum_{j=0}^{\infty}\left(\begin{array}{c}
\beta-1 \\
j
\end{array}\right) \frac{(-x)^{j}}{\alpha+j} F_{1}(\alpha+j, \varrho, \lambda, \alpha+j+1 ; u x, v x),
\end{aligned}
$$

where the last step follows by application of equation (2.2.8.5) in Prudnikov et al. (1986, Volume 1). Note that the infinite sum in (6) will reduce to a finite sum if $\beta$ is an integer.

4. Moments. The $n$th moment of a random variable $X$ with pdf (2) can be calculated easily as

$$
\begin{aligned}
\mathrm{E}\left(X^{n}\right) & =C \int_{0}^{1} \frac{x^{\alpha-1}(1-x)^{\beta-1}}{(1-u x)^{\varrho}(1-v x)^{\lambda}} d x \\
& =C B(\alpha+n, \beta) F_{1}(\alpha+n, \varrho, \lambda, \alpha+\beta+n ; u, v),
\end{aligned}
$$

where we have applied equation (2.2.8.5) in Prudnikov et al. (1986, Volume 1). One can also derive four series representations for the $n$th moment similar to those in Section 3. Firstly, using the expansion (3), one can write

$$
\begin{aligned}
\mathrm{E}\left(X^{n}\right)= & C \int_{0}^{1} x^{n+\alpha-1}(1-x)^{\beta-1}(1-u x)^{-\varrho}(1-v x)^{-\lambda} d x \\
= & C \int_{0}^{1} x^{n+\alpha-1}(1-x)^{\beta-1}\left\{\sum_{j=0}^{\infty}\left(\begin{array}{c}
-\varrho \\
j
\end{array}\right)(-u x)^{j}\right\}(1-v x)^{-\lambda} d x \\
= & C \sum_{j=0}^{\infty}\left(\begin{array}{c}
-\varrho \\
j
\end{array}\right)(-u)^{j} \int_{0}^{1} y^{n+j+\alpha-1}(1-x)^{\beta-1}(1-v x)^{-\lambda} d x \\
= & C \sum_{j=0}^{\infty}\left(\begin{array}{c}
-\varrho \\
j
\end{array}\right)(-u)^{j} B(n+\alpha+j, \beta) \\
& \times{ }_{2} F_{1}(n+\alpha+j, \lambda ; n+\alpha+\beta+j ; v),
\end{aligned}
$$


where the last step follows by application of equation (2.2.6.1) in Prudnikov et al. (1986, Volume 1). Similarly, using the expansion (4), one can obtain

$$
\begin{aligned}
\mathrm{E}\left(X^{n}\right)= & C \sum_{j=0}^{\infty}\left(\begin{array}{c}
-\lambda \\
j
\end{array}\right)(-v)^{j} B(n+\alpha+j, \beta) \\
& \times{ }_{2} F_{1}(n+\alpha+j, \varrho ; n+\alpha+\beta+j ; u) .
\end{aligned}
$$

Thirdly, using both expansions (3) and (4), one can write

$$
\begin{aligned}
\mathrm{E}\left(X^{n}\right) & =C \int_{0}^{1} x^{n+\alpha-1}(1-x)^{\beta-1}\left\{\sum_{j=0}^{\infty}\left(\begin{array}{c}
-\varrho \\
j
\end{array}\right)(-u x)^{j}\right\}\left\{\sum_{k=0}^{\infty}\left(\begin{array}{c}
-\lambda \\
k
\end{array}\right)(-v x)^{k}\right\} d x \\
& =C \sum_{j=0}^{\infty} \sum_{k=0}^{\infty}\left(\begin{array}{c}
-\varrho \\
j
\end{array}\right)\left(\begin{array}{c}
-\lambda \\
k
\end{array}\right)(-u)^{j}(-v)^{k} \int_{0}^{1} y^{n+j+k+\alpha-1}(1-x)^{\beta-1} d x \\
& =C \sum_{j=0}^{\infty} \sum_{k=0}^{\infty}\left(\begin{array}{c}
-\varrho \\
j
\end{array}\right)\left(\begin{array}{c}
-\lambda \\
k
\end{array}\right)(-u)^{j}(-v)^{k} B(n+\alpha+i+j, \beta),
\end{aligned}
$$

where the last step follows from the definition of the beta function. Finally, using the series expansion (5), one can write

$$
\begin{aligned}
\mathrm{E}\left(X^{n}\right)= & C \int_{0}^{1} x^{n+\alpha-1}\left\{\sum_{j=0}^{\infty}\left(\begin{array}{c}
\beta-1 \\
j
\end{array}\right)(-x)^{j}\right\}(1-u x)^{-\varrho}(1-v x)^{-\lambda} d x \\
= & C \sum_{j=0}^{\infty}\left(\begin{array}{c}
\beta-1 \\
j
\end{array}\right)(-1)^{j} \int_{0}^{1} x^{n+j+\alpha-1}(1-u x)^{-\varrho}(1-v x)^{-\lambda} d x \\
= & C \sum_{j=0}^{\infty}\left(\begin{array}{c}
\beta-1 \\
j
\end{array}\right) \frac{(-1)^{j}}{n+\alpha+j} \\
& \times F_{1}(n+\alpha+j, \varrho, \lambda, n+\alpha+j+1 ; u, v),
\end{aligned}
$$

where the last step follows by application of equation (2.2.8.5) in Prudnikov et al. (1986, Volume 1). Note that the infinite sum in (8) will reduce to a finite sum if $\beta$ is an integer.

5. Mean deviations. The amount of scatter in a population is evidently measured to some extent by the totality of deviations from the mean and the median. These are known as the mean deviation about the mean and the mean deviation about the median, defined by

$$
\delta_{1}(X)=\int_{0}^{1}|x-\mu| f(x) d x \quad \text { and } \quad \delta_{2}(X)=\int_{0}^{1}|x-M| f(x) d x,
$$

respectively, where $\mu=\mathrm{E}(X)$ and $M=\operatorname{Median}(X)$. These measures can be 
calculated using the relationships

$$
\begin{aligned}
\delta_{1}(X) & =\int_{0}^{\mu}(\mu-x) f(x) d x+\int_{\mu}^{1}(x-\mu) f(x) d x \\
& =2 \int_{\mu}^{1}(x-\mu) f(x) d x \\
& =2 \int_{\mu}^{1} x f(x) d x-2 \mu\{1-F(\mu)\} \\
& =2 \mathrm{E}(X)-2 \int_{0}^{\mu} x f(x) d x-2 \mu\{1-F(\mu)\}
\end{aligned}
$$

and

(9)

$$
\begin{aligned}
\delta_{2}(X) & =\int_{0}^{M}(M-x) f(x) d x+\int_{M}^{1}(x-M) f(x) d x \\
& =M F(M)-M\{1-F(M)\}-\int_{0}^{M} x f(x) d x+\int_{M}^{1} x f(x) d x \\
& =2 \int_{0}^{M} x f(x) d x-\mathrm{E}(X) .
\end{aligned}
$$

Thus, calculating $\delta_{1}(X)$ and $\delta_{2}(X)$ amounts to calculating $\int_{0}^{a} x f(x) d x$. Applying the series expansions (3)-(5) in the same way as in Sections 3 and 4, one can obtain the four representations:

$$
\begin{aligned}
\int_{0}^{a} x f(x) d x= & C a^{\alpha+1} \sum_{j=0}^{\infty}\left(\begin{array}{c}
-\varrho \\
j
\end{array}\right) \frac{(-u a)^{j}}{\alpha+j+1} \\
& \times F_{1}(\alpha+j+1,1-\beta, \lambda, \alpha+j+2 ; a, v a),
\end{aligned}
$$

$$
\begin{aligned}
\int_{0}^{a} x f(x) d x= & C a^{\alpha+1} \sum_{j=0}^{\infty}\left(\begin{array}{c}
-\lambda \\
j
\end{array}\right) \frac{(-v a)^{j}}{\alpha+j+1} \\
& \times F_{1}(\alpha+j+1,1-\beta, \varrho, \alpha+j+2 ; a, u a),
\end{aligned}
$$

$$
\begin{aligned}
\int_{0}^{a} x f(x) d x= & C \sum_{j=0}^{\infty} \sum_{k=0}^{\infty}\left(\begin{array}{c}
-\varrho \\
j
\end{array}\right)\left(\begin{array}{c}
-\lambda \\
k
\end{array}\right)(-u)^{j}(-v)^{k} \\
& \times B_{a}(\alpha+i+j+1, \beta),
\end{aligned}
$$

$$
\begin{aligned}
\int_{0}^{a} x f(x) d x= & C a^{\alpha+1} \sum_{j=0}^{\infty}\left(\begin{array}{c}
\beta-1 \\
j
\end{array}\right) \frac{(-x)^{j}}{\alpha+j+1} \\
& \times F_{1}(\alpha+j+1, \varrho, \lambda, \alpha+j+2 ; u a, v a) .
\end{aligned}
$$


Expressions for the mean deviations follow by substituting (10)-(13) into (8) and (9).

6. Rényi entropy. An entropy of a random variable $X$ is a measure of variation of the uncertainty. Rényi entropy is defined by

$$
\mathcal{J}_{R}(\gamma)=\frac{1}{1-\gamma} \log \left\{\int f^{\gamma}(x) d x\right\},
$$

where $\gamma>0$ and $\gamma \neq 1$ (Rényi, 1961). It follows easily by application of equation (2.2.8.5) in Prudnikov et al. (1986, Volume 1) that

$$
\begin{aligned}
\int_{0}^{1} f^{\gamma}(x) d x= & C^{\gamma} \int_{0}^{1} x^{\gamma \alpha-\gamma}(1-x)^{\gamma \beta-\gamma}(1-u x)^{-\gamma \varrho}(1-v x)^{-\gamma \lambda} d x \\
= & C^{\gamma} B(\alpha \gamma-\gamma+1, \beta \gamma-\gamma+1) \\
& \times F_{1}(\alpha \gamma-\gamma+1, \varrho \gamma, \lambda \gamma, \alpha \gamma+\beta \gamma-2 \gamma+2 ; u, v) .
\end{aligned}
$$

Thus, Rényi entropy for (2) is given by

$$
\begin{aligned}
\mathcal{J}_{R}(\gamma)= & \frac{1}{1-\gamma}\{\gamma \log C+\log B(\alpha \gamma-\gamma+1, \beta \gamma-\gamma+1) \\
& \left.+\log F_{1}(\alpha \gamma-\gamma+1, \varrho \gamma, \lambda \gamma, \alpha \gamma+\beta \gamma-2 \gamma+2 ; u, v)\right\} .
\end{aligned}
$$

7. Asymptotics. If $X_{1}, \ldots, X_{n}$ is a random sample from (2) and if $\bar{X}=$ $\left(X_{1}+\cdots+X_{n}\right) / n$ denotes the sample mean then by the usual central limit theorem $\sqrt{n}(\bar{X}-\mathrm{E}(X)) / \sqrt{\operatorname{Var}(X)}$ approaches the standard normal distribution as $n \rightarrow \infty$. Sometimes one would be interested in the asymptotics of the extreme values $M_{n}=\max \left(X_{1}, \ldots, X_{n}\right)$ and $m_{n}=\min \left(X_{1}, \ldots, X_{n}\right)$. Note from (2) that $f(t) \sim C t^{\alpha-1}$ as $t \rightarrow 0$ and $f(t) \sim C(1-u)^{-\varrho}(1-v)^{-\lambda}(1-t)^{\beta-1}$ as $t \rightarrow 1$. Thus, it follows by using L'Hospital's rule that

$$
\frac{1-F(1-x h)}{1-F(1-h)} \rightarrow x^{\beta} \text { and } \quad \frac{F(x h)}{F(h)} \rightarrow x^{\alpha}
$$

as $h \rightarrow 0$. Hence, it follows from Theorem 1.6.2 in Leadbetter et al. (1987) that there must be norming constants $a_{n}>0, b_{n}, c_{n}>0$ and $d_{n}$ such that

$$
\operatorname{Pr}\left\{a_{n}\left(M_{n}-b_{n}\right) \leq x\right\} \rightarrow \exp \left\{-(-x)^{\beta}\right\}
$$

and

$$
\operatorname{Pr}\left\{c_{n}\left(m_{n}-d_{n}\right) \leq x\right\} \rightarrow 1-\exp \left(-x^{\alpha}\right)
$$

as $n \rightarrow \infty$.

8. Estimation. Here, we consider maximum likelihood estimation of the parameters when $X_{1}, \ldots, X_{n}$ is a random sample from (2) and also provide expressions for the associated Fisher information matrix. The log- 
likelihood is

$$
\begin{aligned}
\log L(\alpha, \beta, \varrho, \lambda, u, v)= & n \log C+(\alpha-1) \sum_{j=1}^{n} \log X_{j}+(\beta-1) \sum_{j=1}^{n} \log \left(1-X_{j}\right) \\
& -\varrho \sum_{j=1}^{n} \log \left(1-u X_{j}\right)-\lambda \sum_{j=1}^{n}\left(1-v X_{j}\right) .
\end{aligned}
$$

The first derivatives with respect to the six parameters are:

$$
\begin{aligned}
& \frac{\partial \log L}{\partial \alpha}=\sum_{j=1}^{n} \log X_{j}+\frac{n}{C} \frac{\partial C}{\partial \alpha}, \\
& \frac{\partial \log L}{\partial \beta}=\sum_{j=1}^{n} \log \left(1-X_{j}\right)+\frac{n}{C} \frac{\partial C}{\partial \beta}, \\
& \frac{\partial \log L}{\partial \varrho}=-\sum_{j=1}^{n} \log \left(1-u X_{j}\right)+\frac{n}{C} \frac{\partial C}{\partial \varrho}, \\
& \frac{\partial \log L}{\partial z}=-\sum_{j=1}^{n} \log \left(1-v X_{j}\right)+\frac{n}{C} \frac{\partial C}{\partial \lambda}, \\
& \frac{\partial \log L}{\partial u}=\varrho \sum_{j=1}^{n} \frac{X_{j}}{1-u X_{j}}+\frac{n}{C} \frac{\partial C}{\partial u}, \\
& \frac{\partial \log L}{\partial v}=\lambda \sum_{j=1}^{n} \frac{X_{j}}{1-v X_{j}}+\frac{n}{C} \frac{\partial C}{\partial v} .
\end{aligned}
$$

Thus, the maximum likelihood estimates of the six parameters are the solutions of the equations:

$$
\begin{aligned}
\frac{n}{C} \frac{\partial C}{\partial \alpha} & =-\sum_{j=1}^{n} \log X_{j}, \\
\frac{n}{C} \frac{\partial C}{\partial \beta} & =-\sum_{j=1}^{n} \log \left(1-X_{j}\right), \\
\frac{n}{C} \frac{\partial C}{\partial \varrho} & =\sum_{j=1}^{n} \log \left(1-u X_{j}\right), \\
\frac{n}{C} \frac{\partial C}{\partial \lambda} & =\sum_{j=1}^{n} \log \left(1-v X_{j}\right), \\
\frac{n}{C} \frac{\partial C}{\partial u} & =-\varrho \sum_{j=1}^{n} \frac{X_{j}}{1-u X_{j}} \\
\frac{n}{C} \frac{\partial C}{\partial v} & =-\lambda \sum_{j=1}^{n} \frac{X_{j}}{1-v X_{j}}
\end{aligned}
$$


Calculation of the associated Fisher information matrix requires secondorder derivatives of $\log L$. All of the second-order derivatives take the form

$$
\frac{\partial^{2} \log L}{\partial \theta_{i} \partial \theta_{j}}=-\frac{n}{C^{2}} \frac{\partial C}{\partial \theta_{i}} \frac{\partial C}{\partial \theta_{j}}+\frac{n}{C} \frac{\partial^{2} C}{\partial \theta_{i} \partial \theta_{j}}
$$

except for

$$
\begin{aligned}
& \frac{\partial^{2} \log L}{\partial \varrho \partial u}=\sum_{j=1}^{n} \frac{X_{j}}{1-u X_{j}}-\frac{n}{C^{2}} \frac{\partial C}{\partial \varrho} \frac{\partial C}{\partial u}+\frac{n}{C} \frac{\partial^{2} C}{\partial \varrho \partial u}, \\
& \frac{\partial^{2} \log L}{\partial^{2} u}=-\varrho \sum_{j=1}^{n} \frac{X_{j}^{2}}{\left(1-u X_{j}\right)^{2}}-\frac{n}{C^{2}}\left(\frac{\partial C}{\partial u}\right)^{2}+\frac{n}{C} \frac{\partial^{2} C}{\partial u^{2}}, \\
& \frac{\partial^{2} \log L}{\partial \lambda \partial v}=\sum_{j=1}^{n} \frac{X_{j}}{1-v X_{j}}-\frac{n}{C^{2}} \frac{\partial C}{\partial \lambda} \frac{\partial C}{\partial v}+\frac{n}{C} \frac{\partial^{2} C}{\partial \lambda \partial v}, \\
& \frac{\partial^{2} \log L}{\partial^{2} v}=-\lambda \sum_{j=1}^{n} \frac{X_{j}^{2}}{\left(1-v X_{j}\right)^{2}}-\frac{n}{C^{2}}\left(\frac{\partial C}{\partial v}\right)^{2}+\frac{n}{C} \frac{\partial^{2} C}{\partial v^{2}} .
\end{aligned}
$$

Thus, the elements of the Fisher information matrix are straightforward upon noting that

$$
\mathrm{E}\left[X^{n}(1-u X)^{-n}\right]=C B(\alpha+n, \beta) F_{1}(\alpha+n, \varrho+n, \lambda, \alpha+\beta+n ; u, v)
$$

and

$$
\mathrm{E}\left[X^{n}(1-v X)^{-n}\right]=C B(\alpha+n, \beta) F_{1}(\alpha+n, \varrho, \lambda+n, \alpha+\beta+n ; u, v),
$$

which follow by the use of equation (2.2.8.5) in Prudnikov et al. (1986, Volume 1).

9. Application. We now illustrate an application of the proposed generalized beta distributions to consumer price index data. We collected the data on this index for the six countries: United States, United Kingdom, Japan, Canada, Germany and Australia. The data were extracted from the web-site http://www.globalfindata.com/ and the range of data for each country is shown in the table below.

\begin{tabular}{lc}
\hline Country & Range of data \\
\hline Australia & 1901 to 2003 \\
Canada & 1910 to 2003 \\
Germany & 1923 to 2003 \\
Japan & 1868 to 2003 \\
United Kingdom & 1800 to 2003 \\
United States & 1820 to 2003 \\
\hline
\end{tabular}


Taking the ratio $W=X /(X+Y)$, we attempted to model the relative economic performance of each country against another over the range of overlapping years. This yields 15 data sets for the variable $W$. As expected, some of the data for $W$ appeared to concentrate to a subinterval of $[0,1]$ and so suitable location-scale transformations were applied to make the data span from 0 to 1 . For each data set, we fitted the standard beta distribution and the $F_{1}$ beta distribution (given by (1) and (2), respectively) by the method of maximum likelihood. The quasi-Newton algorithm $\mathrm{nlm}$ in the $\mathrm{R}$ software package (Dennis and Schnabel, 1983; Schnabel et al., 1985; Ihaka and Gentleman, 1996) was used to maximize the likelihood.

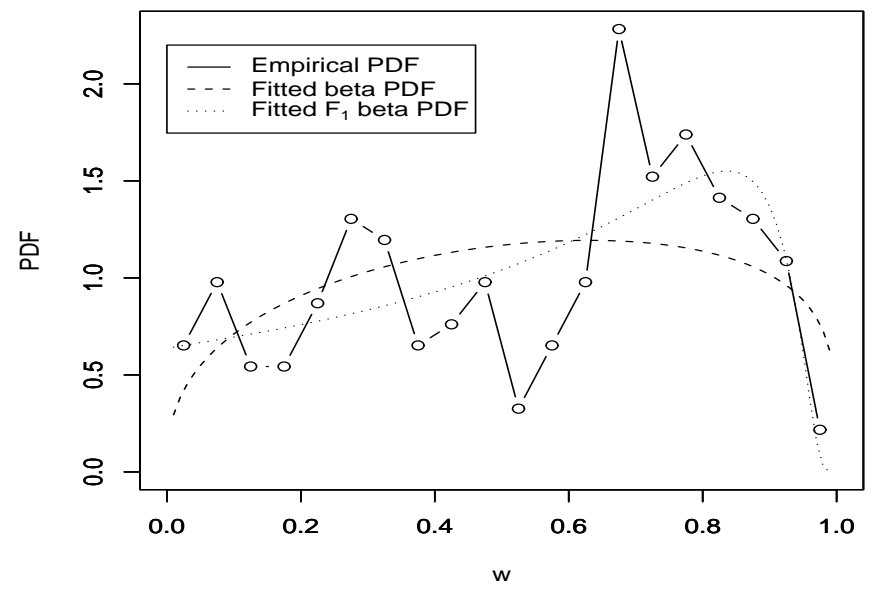

Fig. 4. The empirical and fitted densities for the consumer price indices of the United States and the United Kingdom

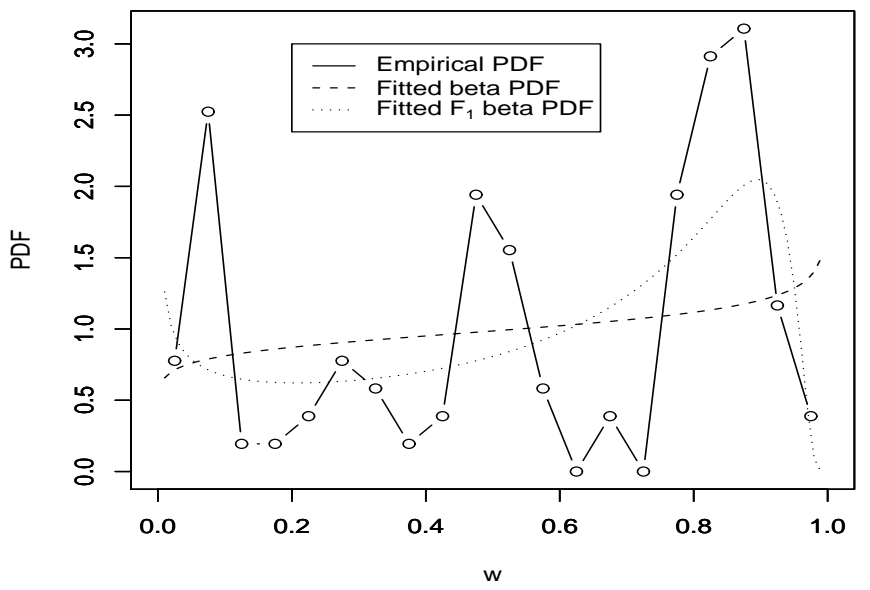

Fig. 5. The empirical and fitted densities for the consumer price indices of the United States and Australia 
The results of the fits were remarkable. In each fit, the maximized loglikelihood for the $F_{1}$ beta model turned up significantly higher than that for the standard beta model. Here, we give details for just two of the 15 data sets:

- for the (United States, United Kingdom) data set the fitted estimates were $\widehat{\alpha}=1.392, \widehat{\beta}=1.230$ with $\log L=5.145$ for the standard beta model and $\widehat{\alpha}=1.003, \widehat{\beta}=32.100, \widehat{u}=0.995, \widehat{v}=0.995, \widehat{\varrho}=16.581$, $\widehat{\lambda}=15.427$ with $\log L=13.150$ for the $F_{1}$ beta model. The corresponding fitted densities superimposed with the empirical density are shown in Figure 3.

- for the (United States, Australia) data set the fitted estimates were $\widehat{\alpha}=1.088, \widehat{\beta}=0.909$ with $\log L=1.372$ for the standard beta model and $\widehat{\alpha}=0.675, \widehat{\beta}=37.710, \widehat{u}=0.996, \widehat{v}=0.996, \widehat{\varrho}=19.146, \widehat{\lambda}=$ 18.992 with $\log L=11.518$ for the $F_{1}$ beta model. The corresponding fitted densities superimposed with the empirical density are shown in Figure 4.

So, we can conclude at least in this situation that the $F_{1}$ beta model is better than one based on the standard beta distribution.

\section{References}

C. Armero and M. J. Bayarri (1994), Prior assessments for prediction in queues, The Statistician 43, 139-153.

J. E. Dennis and R. B. Schnabel (1983), Numerical Methods for Unconstrained Optimization and Nonlinear Equations, Prentice-Hall, Englewood Cliffs, NJ.

I. S. Gradshteyn and I. M. Ryzhik (2000), Table of Integrals, Series, and Products, 6th ed., Academic Press, San Diego, CA.

A. K. Gupta and S. Nadarajah (2004), Handbook of Beta Distribution and Its Applications, Dekker, New York.

R. Ihaka and R. Gentleman (1996), A language for data analysis and graphics, J. Comput. Graph. Statist. 5, 299-314.

N. L. Johnson, S. Kotz and N. Balakrishnan (1995), Continuous Univariate Distributions, Volume 2, 2nd ed., Wiley, New York.

M. R. Leadbetter, G. Lindgren and H. Rootzén (1987), Extremes and Related Properties of Random Sequences and Processes, Springer, New York.

D. L. Libby and M. R. Novick (1982), Multivariate generalized beta-distributions with applications to utility assessment, J. Educational Statistics 7, 271-294.

A. P. Prudnikov, Y. A. Brychkov and O. I. Marichev (1986), Integrals and Series, Volumes 1-3, Gordon and Breach, Amsterdam.

A. Rényi (1961), On measures of entropy and information, in: Proc. 4th Berkeley Symposium on Mathematical Statistics and Probability, Vol. I, Univ. of California Press, Berkeley, CA, 547-561. 
R. B. Schnabel, O. I. Koontz and B. E. Weiss (1985), A modular system of algorithms for unconstrained minimization, ACM Trans. Math. Software 11, 419-440.

School of Mathematics

University of Manchester

Manchester M60 1QD, UK

E-mail: saralees.nadarajah@manchester.ac.uk
Department of Engineering Management and Systems Engineering

George Washington University Washington, DC 20052, U.S.A.

Received on 28.6.2006 